\title{
Key Considerations in the Modeling of Tropical Maritime Microwave Attenuations
}

\author{
Yee Hui Lee ${ }^{1}$ and Yu Song Meng ${ }^{2}$ \\ ${ }^{1}$ School of Electrical and Electronic Engineering, Nanyang Technological University, 50 Nanyang Avenue, Singapore 639798 \\ ${ }^{2}$ National Metrology Centre, Agency for Science, Technology and Research (A*STAR), 1 Science Park Drive, Singapore 118221
}

Correspondence should be addressed to Yu Song Meng; ysmeng@ieee.org

Received 3 December 2014; Accepted 5 March 2015

Academic Editor: Yuan Yao

Copyright ( 2015 Y. H. Lee and Y. S. Meng. This is an open access article distributed under the Creative Commons Attribution License, which permits unrestricted use, distribution, and reproduction in any medium, provided the original work is properly cited.

\begin{abstract}
This paper presents some key considerations for modeling of over-sea radio-wave propagations in $5 \mathrm{GHz}$ band. The summarized information is based on a series of measurement campaigns which were recently carried out in the tropical maritime environments near Singapore. Multiray propagations and ducting of radio waves have been highlighted and considered in over-sea path loss modeling and prediction. It is noted that the sea-surface reflection is an important contribution in the received field, while the duct layers could enhance the radio-wave propagations. Our studies also show that the refracted ray inside evaporation duct could be a strong ray for short-range near sea-surface applications and needs to be properly evaluated.
\end{abstract}

\section{Introduction}

Recently, there are some growing interests in deployments of C-band (e.g., $5 \mathrm{GHz}$ for our interest) radio systems in the maritime environments such as high-speed WiMAX at seaports $[1,2]$, microwave landing system in a seashore country $[3,4]$, and military maritime surveillance [5]. Generally, these applications [1-5] require the microwave radio systems to be operated in over-sea line-of-sight (LoS) maritime environments (e.g., between a base station and a moving vessel/ aircraft), except for some scenarios where ships could block the maritime radio links of interest $[6,7]$.

From the literature, there are limited works on maritime propagation loss predictions, related to the above-mentioned applications. For example, ITU-R P.1546 [8] provides some recommendations for propagation loss predictions over sea paths. However, it is only for the operating frequencies between $30 \mathrm{MHz}$ and $3 \mathrm{GHz}$. When the frequency increases above $3 \mathrm{GHz}$ (e.g., $5 \mathrm{GHz}$ in our studies), an evaporation duct over the sea surface could result in a substantial increase in the received signal strength $[9,10]$, although works in [11] reveal that the propagation loss for a $5 \mathrm{GHz}$ over-sea channel can be predicted using free space loss (FSL) model when the transmission range is less than 10 nautical miles $(18.5 \mathrm{~km})$.
However, for a tropical maritime environment, its higher temperature compared to the temperate climate in other areas can result in a higher evaporation rate of the sea water and therefore a larger evaporation duct height [12]. It thereby could start to trap and affect the radio waves at a shorter distance not only for longer distance (e.g., beyond LoS communications in [13]).

Therefore to deploy a reliable microwave radio system in tropical maritime environments, understanding of over-sea radio-wave propagations becomes very important for system designers. A series of measurement campaigns in $5 \mathrm{GHz}$ band were then carried out in the maritime environments near Singapore in the past few years, in order to characterize the LoS over-sea radio channels. In this paper, some interesting findings and observations from these activities will be summarized and presented. It is noted that the reported signal enhancement below is the level by which the measured signal strength exceeds that for free space propagation.

\section{Basic of Radio-Wave Propagations}

LoS radio-wave propagations with a clear air condition are mainly affected by the properties of atmospheric gases. Oxygen and water vapor have been identified as two main 


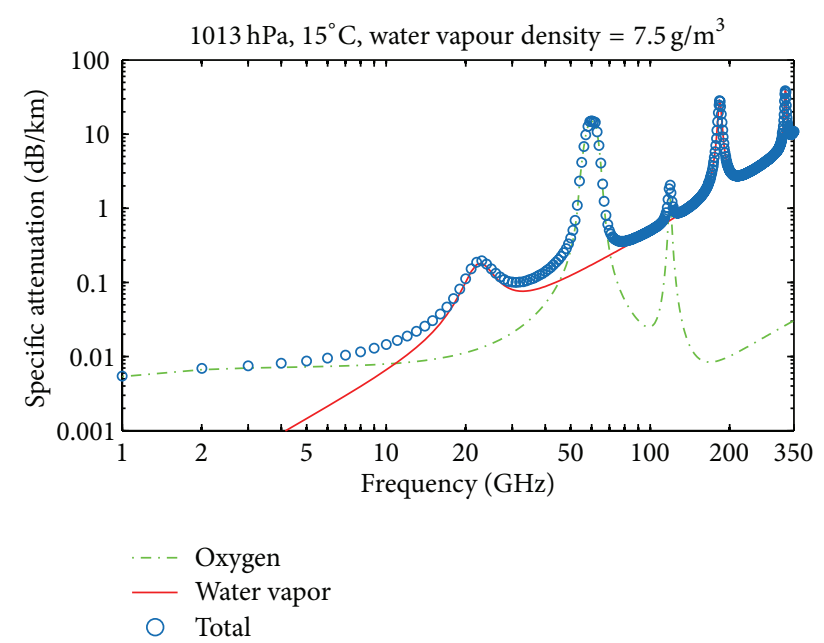

Figure 1: Approximate specific attenuation due to atmospheric gases [14].

sources for the atmospheric attenuation in [14], where specific attenuation curves in $\mathrm{dB} / \mathrm{km}$ at sea level for dry air and water vapor with a density of $7.5 \mathrm{~g} / \mathrm{m}^{3}$ have been recommended from 1 to $350 \mathrm{GHz}$ and shown in Figure 1. It can be found that for a clear air condition atmospheric attenuation on the propagating waves in the $5 \mathrm{GHz}$ band is almost negligible $(<0.01 \mathrm{~dB} / \mathrm{km})$. Hence, the distance dependency of radiowave propagation loss is a key factor we need to consider for $5 \mathrm{GHz}$ radio planning, and will be discussed in this paper.

2.1. Measurements of the Propagation Loss. For measuring the radio-wave propagation loss, typical channel soundings have been introduced in [15]. Basically, the channel measurements require a transmitter and a receiver. Figure 2 presents a very basic setup for radio-wave propagation measurements, using continuous-wave (i.e., narrowband) sounding technique. At the transmitter, the output from a signal generator will be amplified before being sent out from the transmitting antenna. After the signal propagates through the channel of interest (e.g., over-sea channel in our study), the captured signal by a receiving antenna will be filtered and then indicated on a spectrum analyzer. The antennas could be directional or omnidirectional depending on the applications. The propagation loss PL for the setup shown in Figure 2 can be estimated through

$$
\mathrm{PL}=P_{T}+G_{\mathrm{Amp}}+G_{\mathrm{AntT}}+G_{\mathrm{AntR}}-L_{f}-P_{R},
$$

where $P_{T}$ is the output power from signal generator, $G_{\mathrm{Amp}}$ is the gain of amplifier, $G_{\mathrm{Ant} T}$ and $G_{\mathrm{AntR}}$ are the gains of transmitting and receiving antennas, $L_{f}$ is the loss of filter, and $P_{R}$ is the indicated power on spectrum analyzer, respectively. All the parameters shown in (1) are in $\mathrm{dB}$.

It is noted that if wideband information is of interest, coded signal such as spread spectrum technique using maximal-length pseudonoise sequence can be implemented. In such case, vector signal generator at the transmitter can be used with a digitizer at the receiver as we reported in [16].
Other sounding solutions [15] can also be applied depending on the resources available.

2.2. Modeling of the Propagation Loss. Typically for the radiowave propagation in free space with a clear air condition, the propagation loss can be modeled and predicted using the FSL model [15] as follows:

$$
\mathrm{PL}_{\mathrm{FSL}}=-27.56+20 \log _{10}(f)+20 \log _{10}(d),
$$

where $\mathrm{PL}_{\mathrm{FSL}}$ is the FSL in $\mathrm{dB}, f$ is the frequency in $\mathrm{MHz}$, and $d$ is the propagation distance in meter.

For over-sea radio-wave propagation as those reported in $[3,17,18]$, sea-surface reflection is another important component in the received field. Ideally when a reflected ray exists besides the LoS (direct ray) component, the propagation loss could be modeled using a 2-ray path loss model. For neargrazing incidence, the reflection coefficient for a vertically polarized wave approaches -1 . Therefore, the 2-ray path loss model can be simplified as [15]

$$
\mathrm{PL}_{2 \text {-ray }}=-10 \log _{10}\left\{\left(\frac{\lambda}{4 \pi d}\right)^{2}\left[2 \sin \left(\frac{2 \pi h_{t} h_{r}}{\lambda d}\right)\right]^{2}\right\} \text {, }
$$

where $\mathrm{PL}_{2 \text {-ray }}$ is the 2-ray propagation loss in $\mathrm{dB}, \lambda$ is the wavelength in meter, and $h_{t}, h_{r}$ are the heights of a transmitter and a receiver in meter.

However it is sometimes difficult to identify the occurrence of 2-ray propagations; a generic formula has therefore been adopted for modeling the propagation loss $\mathrm{PL}_{G}$ in $\mathrm{dB}$ as $[3,13,19]$

$$
\mathrm{PL}_{G}=A+10 n \log _{10}(d)+X_{f} \text {, }
$$

where $A$ is a constant and normally measured at a reference location from the transmitter, $n$ is the relevant path-loss exponent, and $X_{f}$ is the shadowing factor. For air-to-ground propagations over sea surface, the shadowing could be caused by aircraft maneuvering as we reported in [20]. The path-loss exponent $n$, parameter $A$, and $X_{f}$ in (4) can be extracted from the measured data using the least mean square (LMS) curvefitting technique as in [3].

It is noted that, due to the distance-dependent refractivity conditions and rough sea surfaces [13], the estimated pathloss exponent $n$ is very site-dependent and may not be applicable for other sites. For example, our experimental studies in [3] have indicated that, for the same sea area, the empirically determined path-loss exponent $n$ is within the range of 0.14 to 2.46 with varying the antenna heights and propagation ranges. Therefore, the path loss model (4) has to be restrictedly applied.

In the following, we mainly focus on the implementation of 2-ray or multiray path loss model with considering the duct effects for over-sea radio-wave propagations.

\section{Over-Sea Radio-Wave Propagations}

Generally, over-sea propagation environments can be classified according to the position of radio platforms as being near to the sea surface and hundreds of meters above the sea surface 


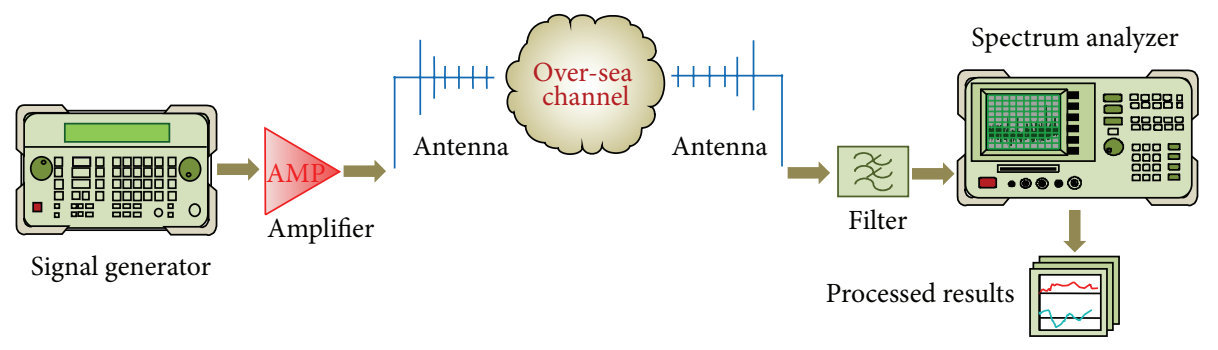

FIGURE 2: Basic idea for propagation loss measurements.

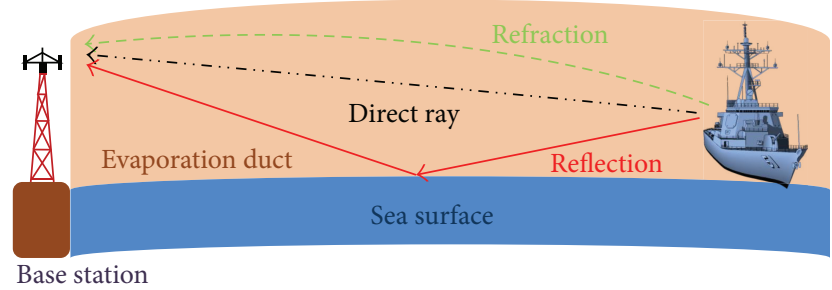

FIGURE 3: Main radio-wave propagation mechanisms for near seasurface applications in an evaporation duct.

or higher. In the following, we will mainly summarize some experimentally evaluated information, based on a series of measurement campaigns which were carried out recently in the tropical maritime environments near Singapore.

Main radio-wave propagation mechanisms for different application scenarios will be presented in detail based on our observations. It is noted that, in our measurement campaigns [3-5], the receiver was located on the shore while the transmitter was placed on a moving vessel/aircraft over the sea surface.

3.1. Near to the Sea Surface. This is an application scenario when the transceiver platform is near to the sea surface, for example, the communications between a ship on sea and an onshore base station as shown in Figure 3. In order to understand the realistic radio-wave propagation mechanisms in such an application environment, we have carried out more than 20 measurement campaigns for proper channel characterizations at $5.15 \mathrm{GHz}$. The measurements were performed over an open sea area off the southeast coast of Singapore, with varying the base station height of $7.6,10$, and $20 \mathrm{~m}$, respectively, and a fixed transmitter height of $3.5 \mathrm{~m}$ approximately above sea level which was on board a speed boat. Some latest results were reported in [5].

Our studies show that, besides the direct ray and the seasurface reflected ray (usually forming 2-ray propagations), the refraction due to the possible evaporation duct as shown in Figure 3 could be another important contribution. The evaporation duct is formed due to the humidity difference in the air-sea boundary and presents up to 90 percent of the time in the equatorial and tropical regions as reported in [13]. Therefore, the influence from the existing evaporation duct needs to be properly characterized and modeled for near seasurface maritime applications.

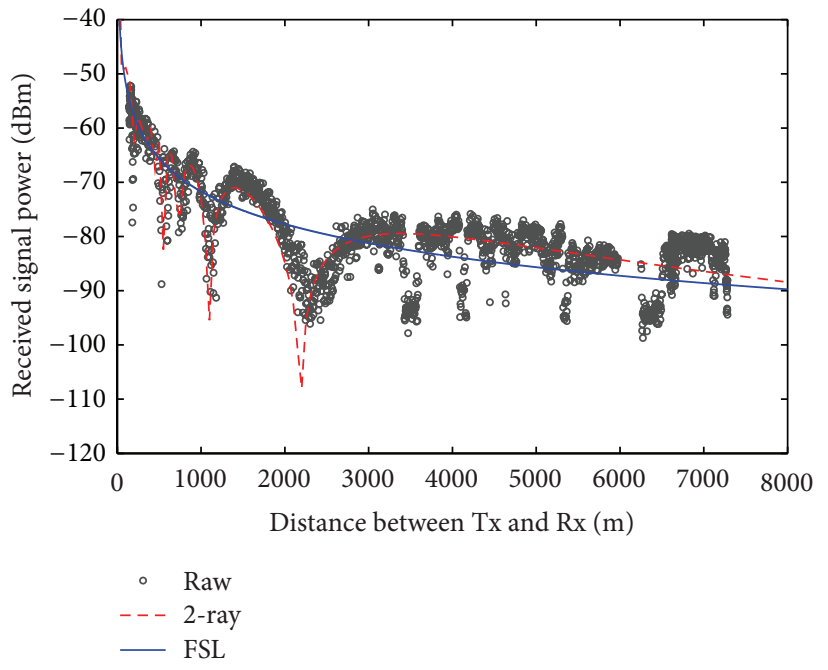

FIGURE 4: Example of the received signal power versus distance with the predicted values using the FSL and 2-ray models [5].

Figure 4 shows a typical example of the measured power versus the transmission distance for near sea-surface radiowave propagations which were performed on November 23, 2011, together with the predicted values using the FSL and 2ray models as in [5]. The reported results in Figure 4 are for a receiver height $h_{r}$ of $20 \mathrm{~m}$ at the base station. From Figure 4 , it can be clearly observed that the FSL model is well suitable for the prediction of local mean (large scale) propagation loss for near sea-surface applications. This observation is very similar to those reported in [11] for a $5-\mathrm{GHz}$ transmitter at $18 \mathrm{~m}$ and a receiver at $30 \mathrm{~m}$ over the sea water. More interestingly, when the propagation distance is less than about $3000 \mathrm{~m}$ in Figure 4, the measured results show a similar trend to the predicated results using the 2-ray path loss model, where the interference nulls are possibly due to the destructive summation of the LoS direct ray and the sea-surface reflected ray.

However, when the propagation distance is beyond $3000 \mathrm{~m}$, there are interference nulls which cannot be predicted using the 2-ray model. Our analysis in [5] indicated that the refraction caused by the evaporation duct as shown in Figure 3 could become significant and cannot be ignored in the modeling process. Therefore, a 3-ray path loss model (including a LoS direct ray, a reflected ray from sea surface, and also a refracted ray caused by evaporation duct) has been evaluated for near sea-surface path loss modeling. 


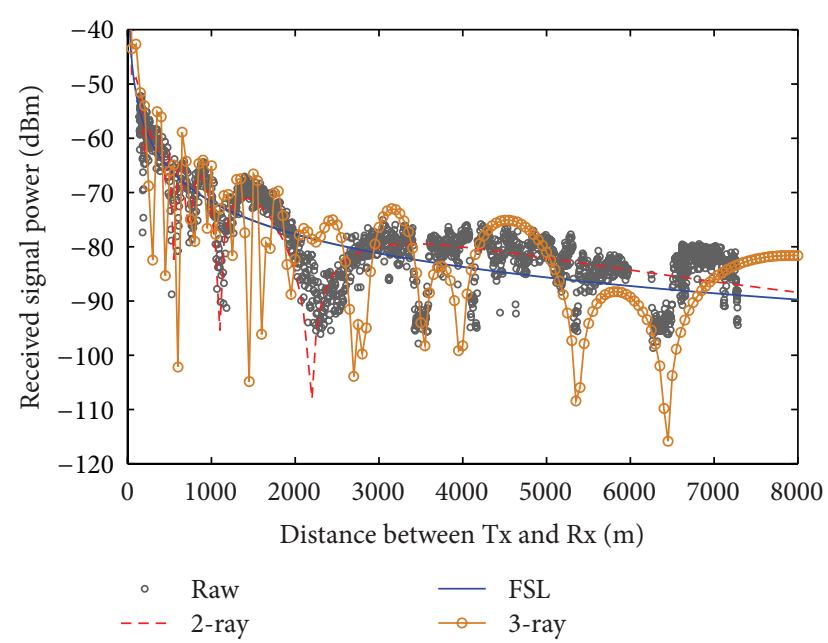

Figure 5: The predicted results using the FSL, 2-ray, and 3-ray models for the received signal strength versus distance in Figure 4.

A simplified 3-ray model [21] has been used with assumption of a horizontally homogeneous evaporation duct layer, a near-grazing incidence on the sea surface, and approximate representation of the refracted wave to reflection:

$$
L_{3 \text {-ray }}(\mathrm{dB})=-10 \log _{10}\left\{\left(\frac{\lambda}{4 \pi d}\right)^{2}[2(1+\Delta)]^{2}\right\},
$$

with

$$
\Delta=2 \sin \left(\frac{2 \pi h_{t} h_{r}}{\lambda d}\right) \sin \left(\frac{2 \pi\left(h_{e}-h_{t}\right)\left(h_{e}-h_{r}\right)}{\lambda d}\right),
$$

where $h_{e}$ is the effective duct height for refraction and can be determined through the curve fitting/regression technique on the measured data as in our previous study [5]. The method is similar to the one reported in [22] where $h_{e}$ was obtained based on a split-step Fourier solution of the parabolic equation approximation to the wave equation.

Figure 5 shows the curve fitting of 3-ray path loss model (5) to the experimental results in Figure 4 for determining $h_{e}$ in the coastal environment near Singapore. From Figure 5, it can be observed that the fitted 3-ray model shows a good prediction ability when the propagation distance is beyond $3000 \mathrm{~m}$ (around a break point for 3-ray propagations to be dominant), which indicates that the third ray (i.e., the highly refracted ray caused by evaporation duct) is significant for near sea-surface radio-wave propagations in our application scenario, and cannot be ignored when the propagation distance increases. The observations hold for more than 20 measurement campaigns performed over the tropical maritime environments under investigations. The empirically estimated effective evaporation duct height $h_{e}$ for refraction is summarized in Figure 6.

It is found that the estimated effective evaporation duct height $h_{e}$ for refraction as shown in Figure 6 is very close to those reported in [23] where the averaged evaporation duct height at a nearby marine environment is between $25 \mathrm{~m}$ and $40 \mathrm{~m}$ during similar months and also very consistent with the observations in [12] as we reported in [5].

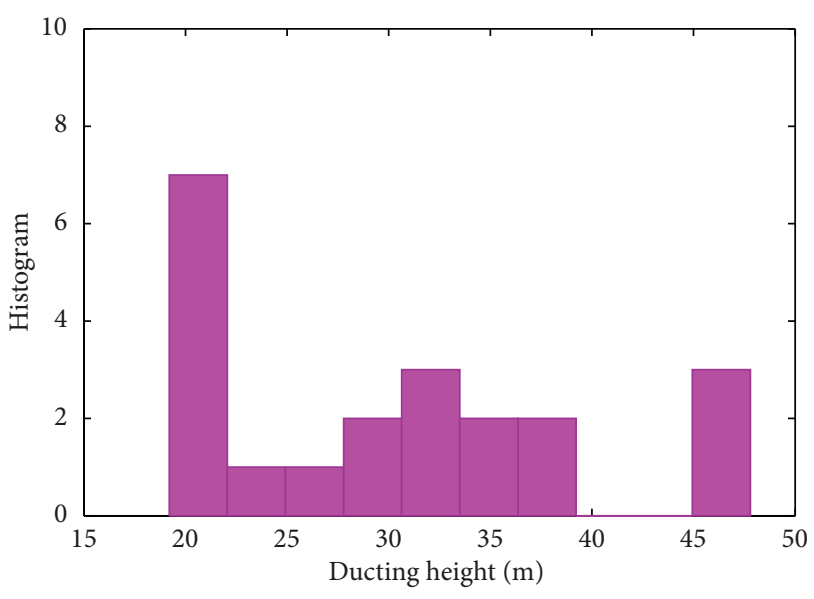

FIGURE 6: Histograms for empirically estimated effective evaporation duct height $h_{e}$ for refraction.

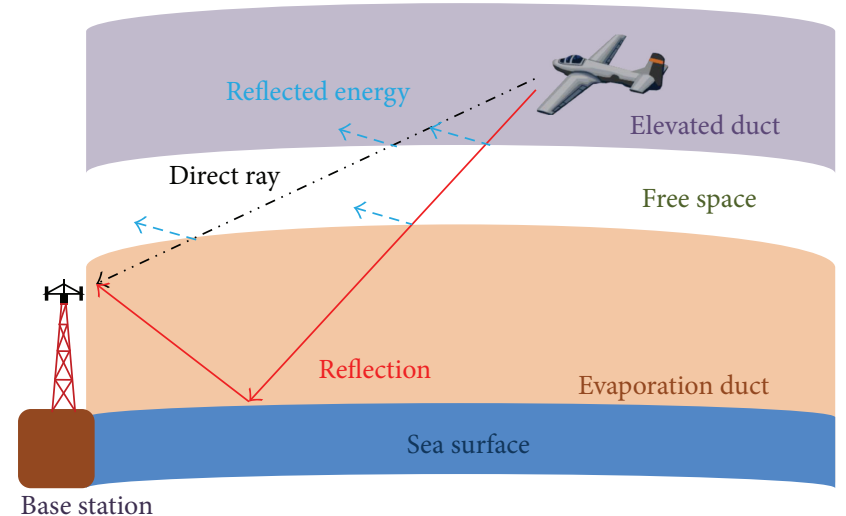

FIGURE 7: Main radio-wave propagation mechanisms for an air-toground over-sea channel.

3.2. Hundreds of Meters above the Sea Surface. This is another application scenario when the transceiver is installed on an airborne platform as shown in Figure 7. Depending on the airborne altitude, the elevated duct in the troposphere could start to affect the air-to-ground over-sea propagations comparing to near sea-surface applications.

It is noted that, in the region around Singapore, the probability of yearly occurrence of elevated duct is more than $10 \%$ of the time and the average yearly elevated duct mean bottom height is more than $1 \mathrm{~km}$ with a mean thickness of around $300 \mathrm{~m}$, according to the statistics derived from 20-year (1977-1996) radiosonde observations as reported in ITU-R P.453 [24].

Sea trials with different measurement configurations have been performed at $5.7 \mathrm{GHz}$ with airborne altitudes of 0.37 , 0.91 , and $1.83 \mathrm{~km}$ and base station heights of $7.65 \mathrm{~m}$ (Channel 1) and $2.10 \mathrm{~m}$ (Channel 2), respectively, at an open space at a coastal area on the eastern side of Singapore. Some results and analysis have been reported in $[3,4]$. The results show that the sea-surface reflected ray is a major component besides the direct ray. As we summarized in [3], the occurrence of sea-surface reflected ray could be more than $40 \%$ of the time. 

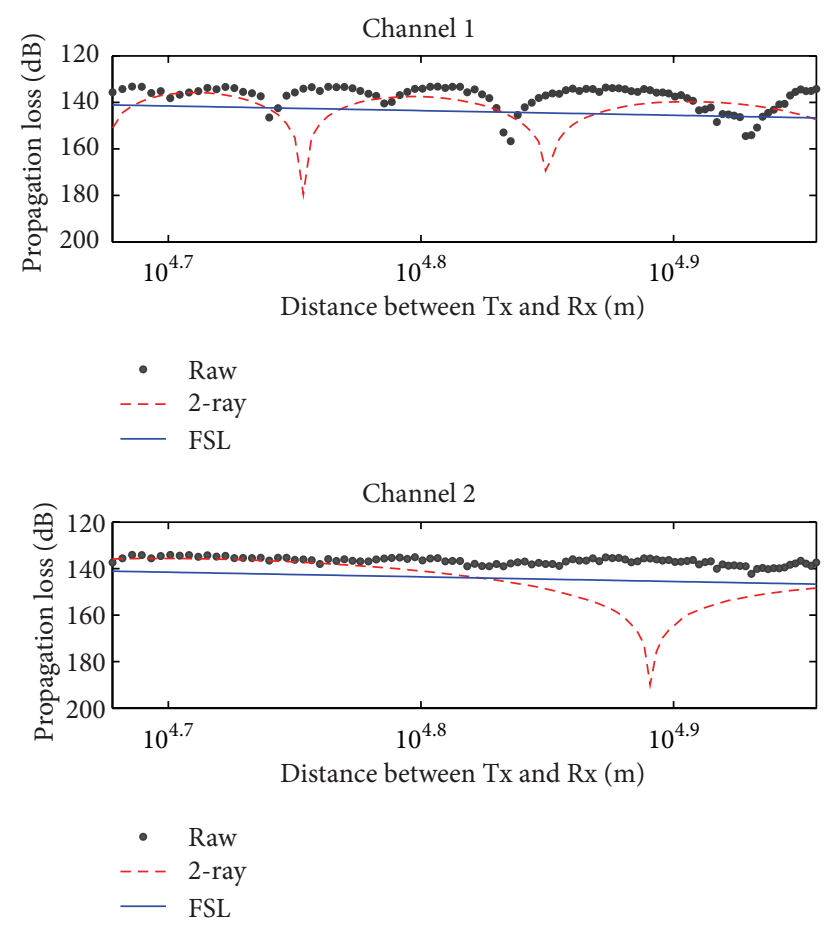

Figure 8: Typical results of propagation loss at airborne altitude of $0.91 \mathrm{~km}[3]$.

An example of the propagation loss at the airborne altitude of $0.91 \mathrm{~km}$ is shown in Figure 8 as a reference. As compared to the near sea-surface transmission which mainly propagates inside an evaporation duct as shown in Figure 3, the airborne transmission needs to propagate through several different layers such as evaporation duct layer, air layer (free space), and elevated duct layer as shown in Figure 7. The resulting different-level reflections could weaken the 2-ray propagation, especially for airborne transmission with much longer propagation range. Therefore, less obvious 2-ray propagations were observed in Figure 8 as compared to the near sea-surface results in Figure 4. It is noted that although there could be 3rd ray, 4th ray, or more [3] (due to multireflections trapped in a duct), their magnitudes are very small referring to the dominant direct ray.

Figure 9 shows a typical example of the measured power delay profile for the over sea-surface airborne channel at $0.91 \mathrm{~km}$ with 2 rays, where the first peak represents the LoS direct ray and the second peak is normally contributed by the sea-surface reflected ray. Clearly the strength of sea-surface reflected ray which is sea-state-dependent is much smaller comparing to the LoS direct ray.

Most importantly for this application scenario, the signal enhancement due to the ducting is very obvious (e.g., there is a much less propagation loss comparing to the free space loss as shown in Figure 8). This is because the propagation range for airborne applications is very long ( $45 \mathrm{~km}$ to $95 \mathrm{~km}$ roughly in the study of this scenario) and thereby results in a significant accumulation of signal enhancement as compared to the shorter near sea-surface application scenario (less than $10 \mathrm{~km}$ in our study).

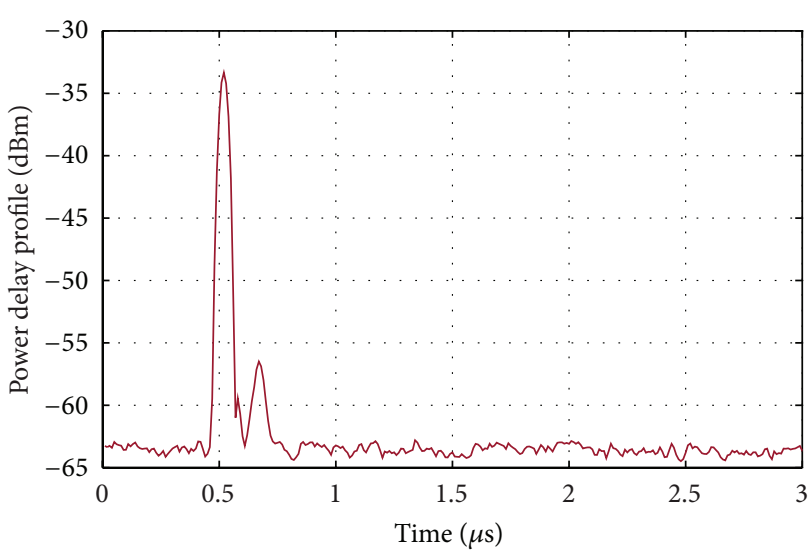

Figure 9: Example of power delay profile for over sea-surface channel at airborne altitude of $0.91 \mathrm{~km}$.
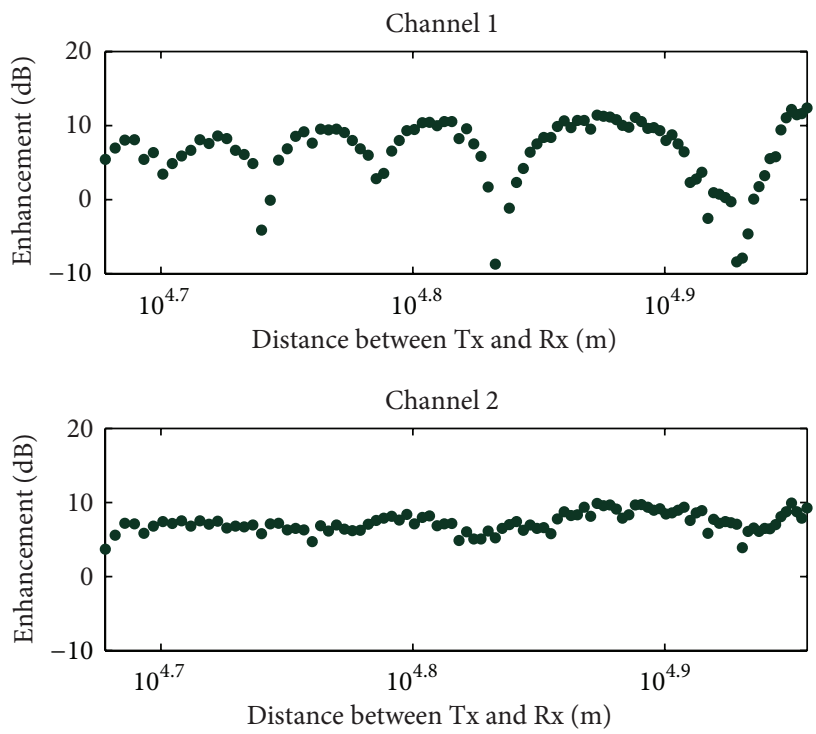

FIGURE 10: Typical results of ducting induced signal enhancement with spatial variations in $\mathrm{dB}$ at airborne altitude of $0.91 \mathrm{~km}$.

The signal enhancement due to the ducting was then estimated referring to the free space loss as reported in [4]. Typical results of ducting induced signal enhancement with spatial variations at airborne altitude of $0.91 \mathrm{~km}$ in $\mathrm{dB}$ are shown in Figure 10. From Figure 10, it is found that as the propagation range increases, the signal enhancement is slightly promoted where some nulls are due to the destructive summation of the direct ray and the sea-surface reflected ray. With the assumptions of homogenous duct layers and no enhancement at distance $d=0 \mathrm{~km}$, the signal enhancement has been linearly modeled with an averaged regression coefficient of $0.1067 \mathrm{~dB} / \mathrm{km}$ [4] based on our measurements with airborne altitudes of $0.37,0.91$, and $1.83 \mathrm{~km}$.

Comparing to the measurement results in [10] where an enhancement in signal strength of more than $10 \mathrm{~dB}$ was observed $48 \%$ of the time along a $27.7 \mathrm{~km}$ over-sea path at $5.6 \mathrm{GHz}$ (i.e., around $0.36 \mathrm{~dB} / \mathrm{km}$ ), the signal enhancement in our study $(\sim 0.11 \mathrm{~dB} / \mathrm{km})$ is relatively small. The main 
reason is that measurements in [10] were performed with the transmitter height of $8.5 \mathrm{~m}$ and the receiver height of $10.5 \mathrm{~m}$ above average tide, and therefore radio-wave propagations mainly occurred within the evaporation duct. For our measurements, they were performed with a transmitter height up to $1.83 \mathrm{~km}$ where the loss at different layer interfaces as shown in Figure 7 could decrease the enhancement effect. Moreover, our signal enhancement $(\sim 0.11 \mathrm{~dB} / \mathrm{km})$ is averaged over the whole propagation path with the free space layer where there is negligible enhancement. Therefore, the smaller enhancement in our study is reasonable.

\section{Conclusions}

This paper summarized some key considerations for modeling of over-sea radio-wave propagations in $5 \mathrm{GHz}$ band, based on a series of measurement campaigns in Singapore.

The application scenarios are classified as near to the sea surface (mainly for ship communications [25]) and hundreds of meters above the sea surface (mainly for airborne maritime applications), depending on the transceiver altitude. Our studies show that the sea-surface reflection is an important contribution for over-sea radio-wave propagations, and its effect is very obvious for near sea-surface applications.

However, the ducts (e.g., evaporation duct and elevated duct) over the sea surface have quite different influences for maritime applications. Our experimental investigations show that there is negligible enhancement of signal strength for short-range near sea-surface applications; however, it could sometimes cause the incident waves to be refracted. The refracted wave may be strong enough and result in unexpected destructive interferences.

For long-range airborne applications, the duct layers can enhance the signal transmissions very significantly. Our studies show that a signal enhancement of $0.1067 \mathrm{~dB} / \mathrm{km}$ could happen for our application scenarios. It is noted that although multiray propagation exists, the strengths of other rays (2nd ray, 3rd ray, etc.) are dependent on the sea state and the condition of ducts and quite often smaller comparing to the direct ray. Therefore, over-sea radio-wave propagation channels need to be properly characterized for a better radio planning.

\section{Conflict of Interests}

The authors declare that there is no conflict of interests regarding the publication of this paper.

\section{Acknowledgments}

This work was supported in part by the Defence Science and Technology Agency, Singapore. The authors would like to thank the engineers from ST Electronics Ltd. and DSO National Laboratories and the propagation team of researchers and students from NTU for their contributions to the experiments.

\section{References}

[1] J. Joe, S. K. Hazra, S. H. Toh et al., "Path loss measurements in sea port for WiMAX," in Proceedings of the IEEE Wireless Communications and Networking Conference (WCNC '07), pp. 1873-1878, March 2007.

[2] J. C. Reyes-Guerrero, M. Bruno, L. A. Mariscal, and A. Medouri, "Buoy-to-ship experimental measurements over sea at $5.8 \mathrm{GHz}$ near urban environments," in Proceedings of the 11th Mediterranean Microwave Symposium (MMS '11), pp. 320-324, September 2011.

[3] Y. S. Meng and Y. H. Lee, "Measurements and characterizations of air-to-ground channel over sea surface at C-band with low airborne altitudes," IEEE Transactions on Vehicular Technology, vol. 60, no. 4, pp. 1943-1948, 2011.

[4] Y. H. Lee and Y. S. Meng, "Empirical modeling of ducting effects on a mobile microwave link over a sea surface," Radioengineering, vol. 21, no. 4, pp. 1054-1059, 2012.

[5] Y. H. Lee, F. Dong, and Y. S. Meng, "Near sea-surface mobile radiowave propagation at $5 \mathrm{GHz}$ : measurements and modeling," Radioengineering, vol. 23, no. 3, pp. 824-830, 2014.

[6] W. Hubert, Y.-M. Le Roux, M. Ney, and A. Flamand, "Impact of ship motions on maritime radio links," International Journal of Antennas and Propagation, vol. 2012, Article ID 507094, 6 pages, 2012.

[7] Y. H. Lee, F. Dong, and Y. S. Meng, "Stand-off distances for non-line-of-sight maritime mobile applications in $5 \mathrm{GHz}$ band," Progress In Electromagnetics Research B, vol. 54, pp. 321-336, 2013.

[8] ITU, "Method for point-to-area predictions for terrestrial services in the frequency range $30 \mathrm{MHz}$ to $3000 \mathrm{MHz}$," ITU Rec. P. 1546-4, ITU, Geneva, Switzerland, 2009.

[9] H. V. Hitney and L. R. Hitney, "Frequency diversity effects of evaporation duct propagation," IEEE Transactions on Antennas and Propagation, vol. 38, no. 10, pp. 1694-1700, 1990.

[10] H. J. M. Heemskerk and R. B. Boekema, "The influence of evaporation duct on the propagation of electromagnetic waves low above the sea surface at 3-94 GHz," in Proceedings of the 8th International Conference on Antennas and Propagation, vol. 1, pp. 348-351, Edinburgh, UK, March-April 1993.

[11] H. V. Hitney, J. H. Richter, R. A. Pappert, K. D. Anderson, and G. B. Baumgartner Jr., "Tropospheric radio propagation assessment," Proceedings of the IEEE, vol. 73, no. 2, pp. 265-283, 1985.

[12] A. Kerans, A. S. Kulessa, E. Lensson, G. French, and G. S. Woods, "Implications of the evaporation duct for microwave radio path design over tropical oceans in Northern Australia," in Proceedings of the Workshop on the Applications of Radio Science, February 2002.

[13] E. Dinc and O. Akan, "Beyond-line-of-sight communications with ducting layer," IEEE Communications Magazine, vol. 52, no. 10, pp. 37-43, 2014.

[14] ITU, "Attenuation by atmospheric gases," ITU Recommendation P.676-10, ITU, Geneva, Switzerland, 2013.

[15] J. D. Parsons, The Mobile Radio Propagation Channel, Wiley, New York, NY, USA, 2nd edition, 2000.

[16] Y. S. Meng and Y. H. Lee, "Practical wideband channel sounding system for air-to-ground measurements at C band," in Proceedings of the IEEE Instrumentation and Measurement Technology Conference (I2MTC '09), pp. 771-775, Singapore, May 2009. 
[17] Q. Lei and M. Rice, "Multipath channel model for over-water aeronautical telemetry," IEEE Transactions on Aerospace and Electronic Systems, vol. 45, no. 2, pp. 735-742, 2009.

[18] D. Matolak, K. Shalkhauser, and R. Kerczewski, "L-band and Cband air-ground channel measurement \& modeling for over-sea conditions," Tech. Rep. ACP WG-F/30 IP 08, ICAO, 2014.

[19] I. J. Timmins and S. O'Young, "Marine communications channel modeling using the finite-difference time domain method," IEEE Transactions on Vehicular Technology, vol. 58, no. 6, pp. 2626-2637, 2009.

[20] Y. S. Meng and Y. H. Lee, "Study of shadowing effect by aircraft maneuvering for air-to-ground communication," $A E U$ : International Journal of Electronics and Communications, vol. 66, no. 1, pp. 7-11, 2012.

[21] W. C. Y. Lee, Mobile Communications Engineering: Theory and Applications, McGraw-Hill, 2nd edition, 1997.

[22] I. Levadnyi, V. Ivanov, and V. Shalyapin, "Assessment of evaporation duct propagation simulation," in Proceedings of the 30th URSI General Assembly and Scientific Symposium (URSIGASS '11), August 2011.

[23] X.-L. Zhao, J.-Y. Huang, and S.-H. Gong, "Statistical analysis of an over-the-sea experimental transhorizon communication at x-band in China," Journal of Electromagnetic Waves and Applications, vol. 22, no. 10, pp. 1430-1439, 2008.

[24] ITU, "The radio refractive index: its formula and refractivity data," ITU Recommendation P.453-10, ITU, Geneva, Switzerland, 2012.

[25] F. Dong, C. W. Chan, and Y. H. Lee, "Channel modeling in maritime environment for USV," in Proceedings of the Defence Technology Asia, Singapore, 2011. 

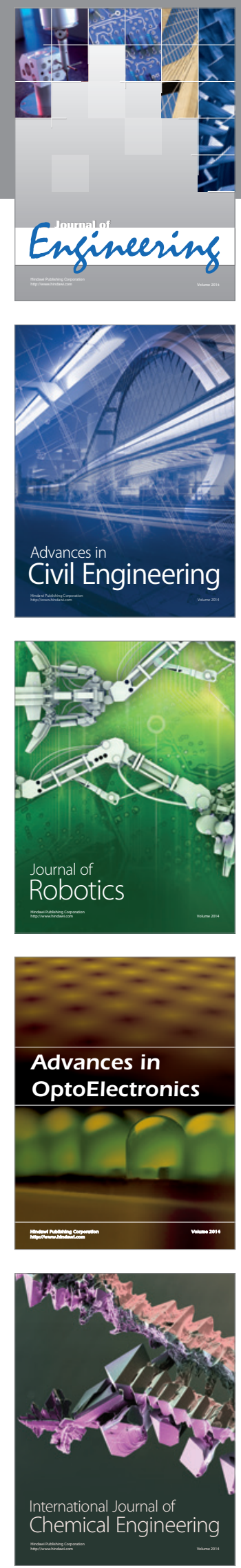

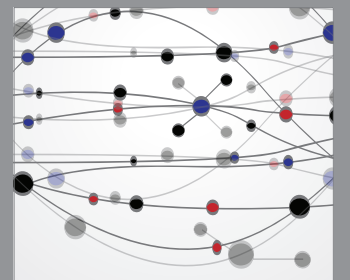

The Scientific World Journal
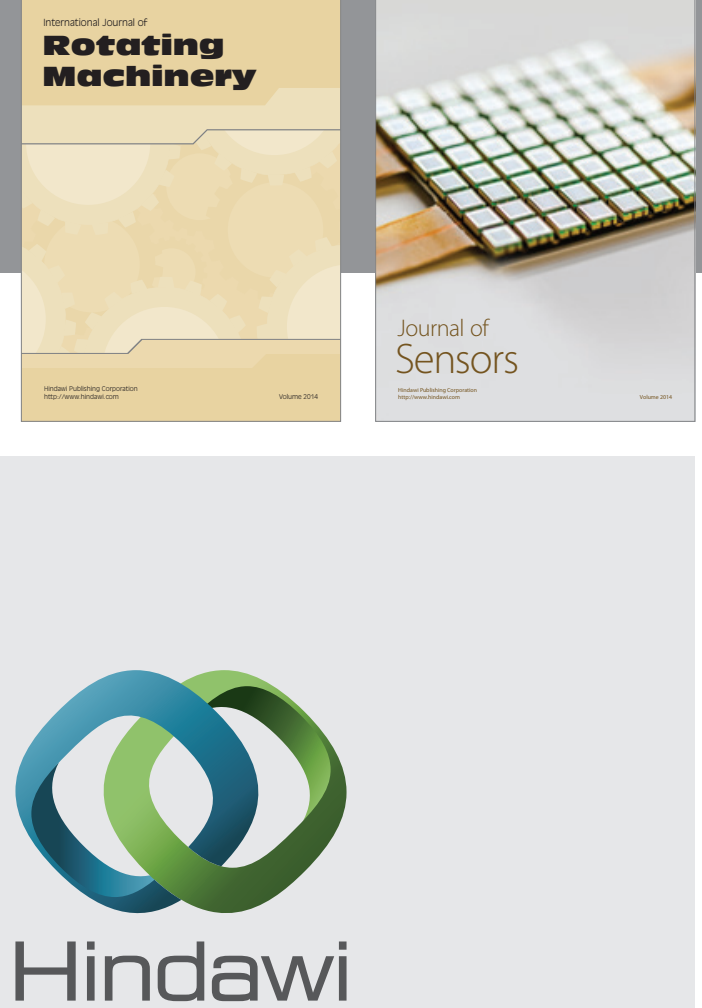

Submit your manuscripts at http://www.hindawi.com
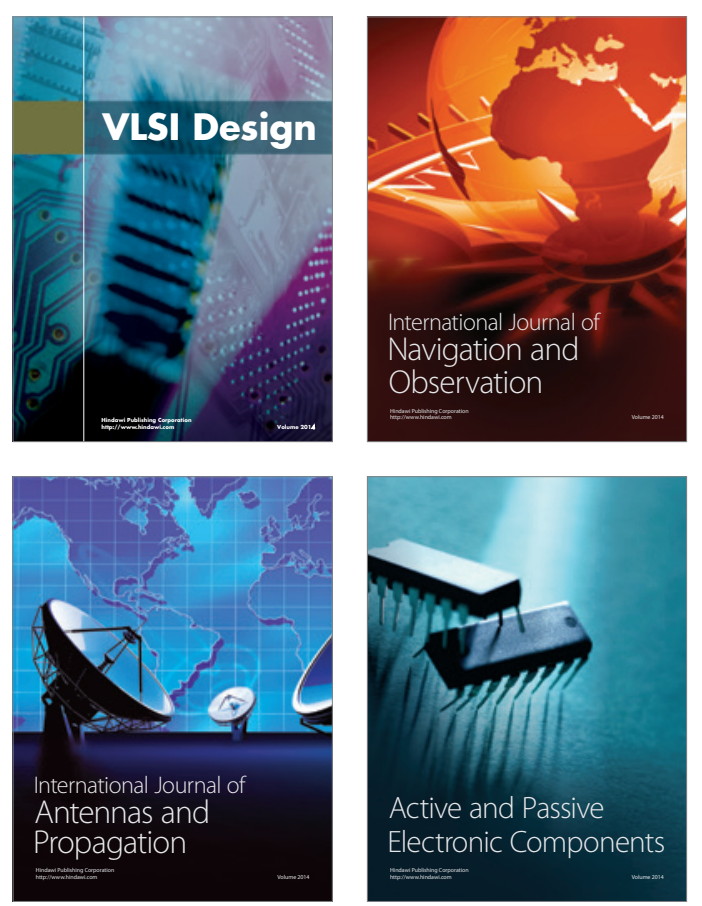
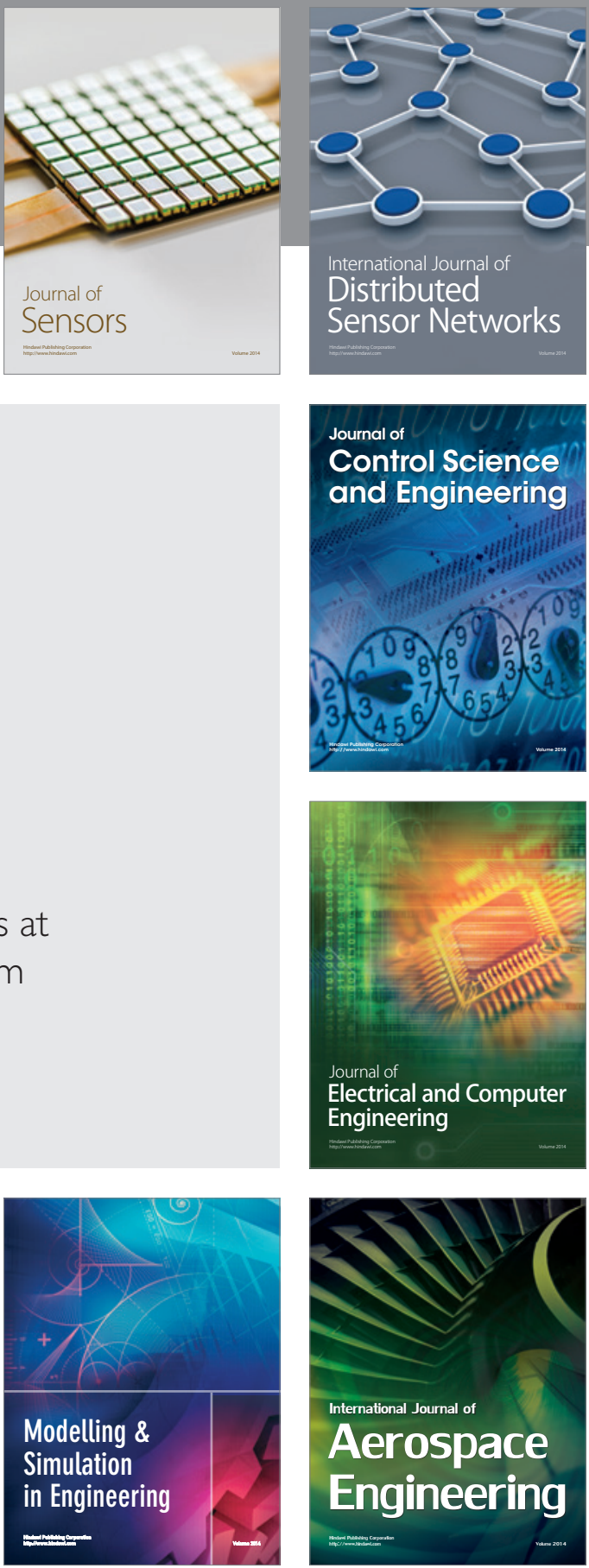

Journal of

Control Science

and Engineering
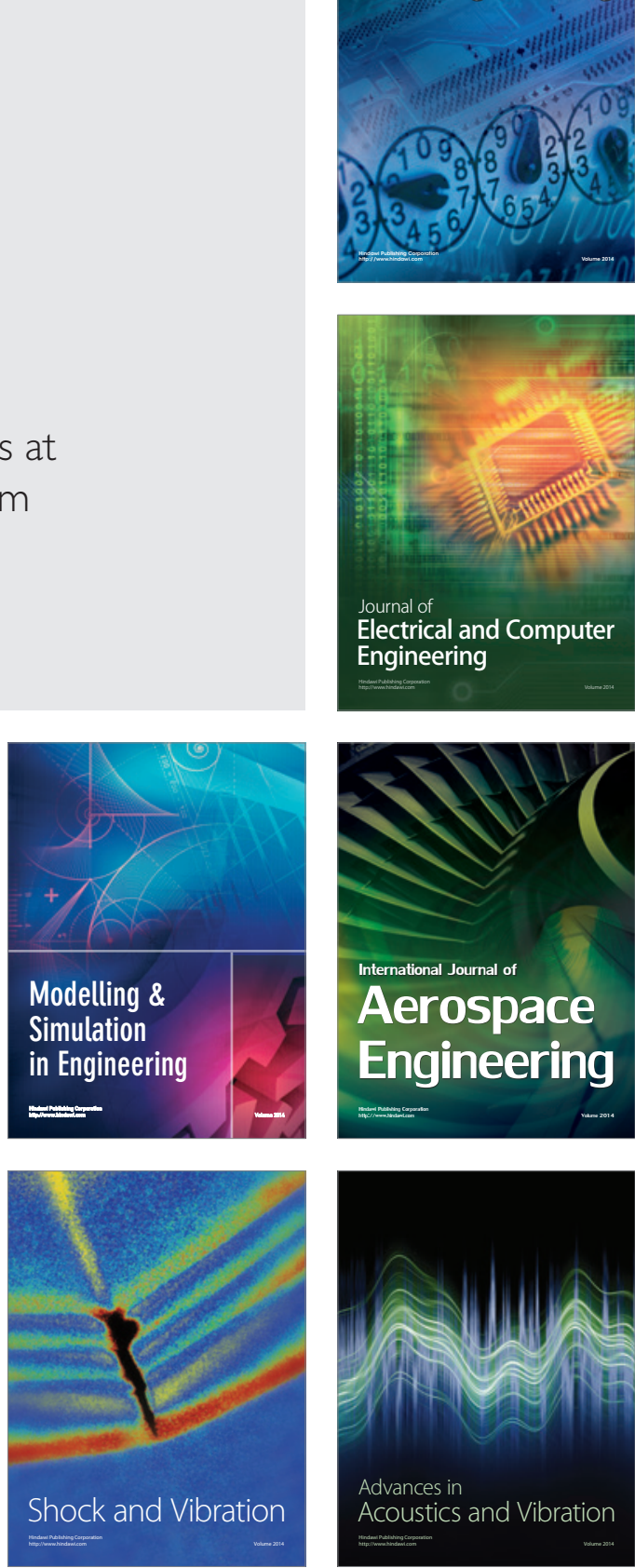